\title{
Einsatz von Taxanen in der Therapie des Mammakarzinoms
}

\section{Taxans in the Treatment of Breast Cancer}

\author{
Gesprächsleitung: Volker Möbus (Frankfurt/M.) \\ Teilnehmer: $\quad$ W. Eiermann, München M. Fridrik, Linz H.-J. Lück, Wiesbaden \\ U. Nitz, Düsseldorf G. Steger, Wien C. Thomssen, Halle (Saale)
}

\section{Frage 1: Beide Taxane haben mit klaren Studiendaten ihren Stellenwert in der adjuvanten Therapie des Mammakarzinoms belegt. Sind Taxane ein Standardregime und welchen Patientengruppen empfehlen Sie eine adjuvante taxanhaltige Therapie?}

Eiermann: Taxane sind heute in Kombination oder Sequenz mit Anthrazyklinen als Standard beim nodal negativen und positiven, sowie beim HER2/neu negativen und positiven Mammakarzinom anzusehen.

Fridrik: Taxane sind kein Standardregime in der adjuvanten Therapie des Mammakarzinoms. Zwei Studien, die Regime mit nur jeweils 2 Substanzen, nämlich Doxorubicin und Cyclophosphamid gegen Doxorubicin und Taxan, verglichen haben, konnten keinen Überlebensgewinn zeigen. Werden mehr als 2 Substanzen verwendet, konnte in den meisten Studien ein Vorteil im Überleben gefunden werden. Am größten ist dieser Überlebensvorteil für Rezeptor-negative, Lymphknoten-positive Tumoren und Patientinnen, die zwischen 51 und 65 Jahre alt sind. Bei letzteren muss aber die Toxizität dieser Therapie beachtet werden.

Lück: Ich empfehle Taxane allen nodal positiven Patientinnen.

Nitz: Ich empfehle bis auf wenige Ausnahmen allen Patientinnen mit Lymphknotenbefall zumindest das PACS-01-Regime oder ein länger dauerndes (8 Kurse) Standardregime. Bei N0Stadien erfolgt eine sorgfältige Abwägung der Risikokonstellation (high risk, z.B. jung, triple negative, G3 usw.). Aufgrund der Datenlage erfolgt hier die Indikationsstellung zurückhaltend.

Steger: Aufgrund der vorliegenden Daten sind die Taxane als etablierter Standard anzusehen, deren Einsatz entsprechend der Studienpopulationen bei Lymphknoten-positiven Patientinnen überlegt werden soll.
Thomssen: Taxanhaltige Chemotherapien sind heutzutage aus der adjuvanten Therapie des Mammakarzinoms nicht mehr wegzudenken. In randomisierten Studien an mehr als 10000 Patientinnen konnte die Wirksamkeit in der adjuvanten Therapie des nodal positiven Mammakarzinoms belegt werden. Insbesondere durch die französische Studie PACS01 und die spanische Studie GEICAM-9906 konnte die Überlegenheit einer Anthrazyklin-Taxan-Sequenz gegenüber der in Europa verbreiteten Standardkombination FEC belegt werden. Für die adjuvante Therapie bei nodal positivem Mammakarzinom sollte daher heutzutage die Sequenz einer Anthrazyklin-Dreierkombination (z.B. FEC), gefolgt von einem Taxan eingesetzt werden. Für das nodal negative Mammakarzinom gibt es dagegen derzeit keine Daten. Eine Indikationsstellung ist in diesem Bereich noch als experimentell anzusehen. Im Rahmen der NNBC 3-Europe-Studie der AGO (Arbeitsgemeinschaft Gynäkologische Onkologie) und GBG (German Breast Group) wird der Einsatz einer Anthrazyklin-Taxan-Sequenz beim high-risk nodal negativen Mammakarzinom überprüft.

\section{Frage 2: Inwieweit ist lhre Entscheidung vom Hormonrezeptorgehalt des Tumors abhängig?}

Eiermann: Docetaxel ist effektiv beim rezeptorpositiven und negativen Mammakarzinom in der Prä- und Postmenopause, vor allem bei 1-3 befallenen Lymphknoten.

Fridrik: Der Einfluss des Hormonrezeptors auf die Wirksamkeit einer adjuvanten taxanhaltigen Therapie ist kontrovers. In einer rezenten retrospektiven Auswertung von 3 CALGBStudien konnte allerdings gezeigt werden, dass sich das Gesamtüberleben durch eine taxanhaltige Chemotherapie bei Östrogenrezeptor-negativen Tumoren um 16,7\% verbessert,

\begin{tabular}{|c|c|c|}
\hline KARGER & (c) 2006 S. Karger GmbH, Freiburg & $\begin{array}{l}\text { Prof. Dr. Volker Möbus } \\
\text { Städtische Kliniken }\end{array}$ \\
\hline $\begin{array}{l}\text { Fax +497614520714 } \\
\text { E-mail Information@Karger.de } \\
\text { www.karger.com }\end{array}$ & $\begin{array}{l}\text { Accessible online at: } \\
\text { www.karger.com/brc }\end{array}$ & $\begin{array}{l}\text { Frauenklinik } \\
\text { Gotenstraße 6-8, 65929 Frankfurt/M., Germany } \\
\text { Tel. +40 69 3106-2339, Fax -3030 } \\
\text { E-Mail MoebusVolk@aol.com }\end{array}$ \\
\hline
\end{tabular}


während bei Östrogenrezeptor-positiven Tumoren die Verbesserung nur $4 \%$ beträgt.

Lück: Aus den Studien mit primär systemischen Therapie wissen wir, dass rezeptornegative Tumoren eine größere Wahrscheinlichkeit für ein Ansprechen haben. Dies gilt für alle Substanzen.

Nitz: Bei N0, Hormonrezeptor-positiven $(\mathrm{HR}+)$ Tumoren erfolgt in Abhängigkeit vom Grading, vom Alter und von der HER2-Überexpression eine alleinige endokrine Therapie oder eine sequentielle chemo-endokrine Therapie. Bei N0 /HR+ bevorzuge ich $6 \times$ FEC als Standard. Bei N+ gebe ich selten, nur bei niedriggradigem Lymphknotenbefall, hohem Rezeptorproteingehalt und älterer Patientin keine Chemotherapie. Ansonsten konsultiere ich gemeinsam mit der Patientin «adjuvantonline.org» und diskutiere den potentiellen Vorteil durch eine Chemotherapie. $6 \times$ FEC scheinen mir nicht verträglicher als $3 \times$ FEC gefolgt von $3 \times$ Docetaxel, so dass ich mich bei Akzeptanz für eine Chemotherapie an dieser Stelle für das taxanhaltige, effektivere Protokoll entscheide.

Steger: Mangels entsprechender klarer Daten kann hier keine valide Empfehlung abgegeben werden, doch sind Patientinnen mit positiven Lymphknoten und hormonunabhängigem Tumor bzw. unsicherer Hormonabhängigkeit sicherlich Kandidatinnen für eine adjuvante Taxantherapie. Bei starker Hormonabhängigkeit kann und muss man auch entsprechend dem sonstigem Risikoprofil andere Therapieoptionen diskutieren.

Thomssen: In einigen Studien scheint die Wirksamkeit der Taxane in der adjuvanten Therapie beim HR- Tumor deutlich besser zu sein, als beim HR+ Tumor. Andere Studien bestätigen diesen prädiktiven Wert des Hormonrezeptorstatus nicht Er spielt daher für die Entscheidung für oder gegen eine taxanhaltige Chemotherapie beim nodal positiven Mammakarzinom nur eine untergeordnete Rolle.

\section{Frage 3: Welchem taxanhaltigen Regime würden Sie in der adjuvanten Situation den Vorzug geben?}

Eiermann: Die Therapieentscheidung fällt entsprechend dem Risikoprofil und dem Nachweis prädiktiver und prognostischer Marker (Zahl der befallenen Lymphknoten, Rezeptorstatus, HER2/neu-Status, Alter der Patientin).

Fridrik: Das Schema sollte neben dem Taxan mindestens 2 weitere Substanzen enthalten. Es ist unentschieden, ob das Taxan gleichzeitig oder sequenziell mit den anderen Substanzen gegeben werden sollte. Unklar ist auch, welchem der beiden Taxane der Vorzug gegeben werden soll. Ich verwende $4 \times$ Doxorubicin/Cyclophosphamid gefolgt von $4 \times$ Paclitaxel.

Lück: Bisher haben sequentielle taxanhaltige Therapien das beste Nutzen/Nebenwirkungsverhältnis aufgewiesen.

Nitz: PACS-01 bei mittlerem Risiko wegen der guten Durchführbarkeit. Und TAC bei hohem Risiko, wegen der vermutlich hohen Wirksamkeit.
Steger: Von den publizierten und zur Verfügung stehenden 3 Therapieschemata $(4 \times \mathrm{AC} \rightarrow 4 \times \mathrm{P}, 6 \times \mathrm{TAC}, 3 \times \mathrm{FEC100} \rightarrow$ $3 \times \mathrm{D})$ erscheint hinsichtlich Risikoreduktion, Toxizitätsprofil und Kosteneffektivität $3 \times$ FEC100 $\rightarrow 3 \times$ D am attraktivsten und hat sich in der Praxis bei uns bereits bewährt. Direkte Vergleichstudien dieser Schemata, aber auch anderer Taxanregime mit den genannten stehen jedoch aus, sodass die Schemawahl individuell getroffen werden muss.

Thomssen: Das derzeit wirksamste taxanfreie Standardregime ist die Dreierkombination FEC (in der kanadischen Version nach Levine 1998 - MA.5 oder der französischen Version nach Bonneterre 2001 - FASG.05) mit einer Epirubicin-Mindestdosierung von $100 \mathrm{mg} / \mathrm{m}^{2}$. Die einzigen taxanhaltigen Regime, die gegen diesen Standard geprüft worden sind und eine höhere Effektivität gezeigt haben, sind das PACS-01-Regime (3 Zyklen FEC gefolgt von 3 Zyklen Docetaxel) und das GEICAM-9906-Regime (4 Zyklen FEC gefolgt $12 \times$ Paclitaxel wöchentlich). Diese beiden Regime würde ich daher bevorzugt in der adjuvanten Therapie des nodal positiven Mammakarzinoms einsetzen. Auch für die docetaxelhaltige Dreierkombination TAC (BCIRG-001) liegen überzeugende Daten vor. Allerdings ist die Toxität dieses Regimes etwas höher als die anderer Regime und der Vergleich mit FAC in der gewählten Dosierung weniger akzeptiert als der Vergleich zu FEC in anderen Studien.

Aufgrund der in San Antonio vorgestellten Daten von Sparano (North American Breast Cancer Intergroup E 1199) wissen wir, dass die Taxane in ihren verschiedenen Applikationsformen keine wesentlichen Unterschiede zeigen. Tendenziell scheint die wöchentliche Paclitaxeltherapie und die 3-wöchentliche Docetaxeltherapie am wirksamsten sein. Die Nebenwirkungsprofile sind sicher in die Entscheidung mit einzubeziehen (Neurotoxizität bei Paclitaxel, Neutropenie bei Docetaxel).

\section{Frage 4: Glauben Sie, dass es einen Wirksamkeits- unterschied in Abhängigkeit von dem gewählten Taxan gibt oder entscheiden Sie sich für ein bestimmtes Regime primär unter Aspekten des Nebenwirkungs- profiles?}

Eiermann:Wie schon erwähnt, gibt es Wirksamkeitsunterschiede, abhängig vom Markerprofil; das Nebenwirkungsprofil ist ebenfalls unbedingt in die Therapieentscheidung mit einzubeziehen.

Fridrik: Derzeit gibt es keine Daten über den direkten Vergleich zwischen Paclitaxel und Docetaxel in der adjuvanten Therapie. Paclitaxel ist deutlich billiger, während Docetaxel eine geringere Neurotoxizität hat. Ich verwende hauptsächlich Paclitaxel.

Lück: Bisher ist in der größten prospektiven randomisierten Studie kein Unterschied erkennbar. Auch in den anderen Studien war der absolute, und damit klinisch relevante Unter- 
schied gering. Das Nebenwirkungspotential von Docetaxel $\left(100 \mathrm{mg} / \mathrm{m}^{2}\right)$ ist aber deutlich erhöht.

Nitz: Nein, ich glaube nicht, dass es einen relevanten Wirksamkeitsvorteil zugunsten einer der Substanzen gibt. Die Entscheidung fällt anhand des Nebenwirkungsprofils.

Steger: Kürzlich publizierte Daten einer Studie, die beide Taxane sowohl als 3-wöchentliches Schema als auch bei wöchentlicher Applikation untersucht hat, konnte keine signifikanten Unterschiede zeigen. Daher bleibt derzeit nur die Entscheidung basierend auf Toxizität und vielleicht Kosteneffektivität.

Frage 5: Vor kurzem sind erstmals zwei Phase-IIIStudien zur wöchentlichen Taxantherapie publiziert worden (North American Breast Cancer Intergroup, E 1199, GEICAM 9906). Erwarten Sie, dass die wöchentliche dosisdichte Gabe eine zunehmende Bedeutung in der adjuvanten Therapie erlangen wird?

Eiermann: Ja.

Fridrik: Am San Antonio Meeting 2005 wurde das North American Breast Cancer Intergroup Trial E1199 vorgestellt. Dies Studie fand weder im krankheitsfreien Überleben noch im Gesamtüberleben einen Unterschied zwischen adjuvantem Paclitaxel und Docetaxel oder zwischen 3-wöchentlicher und wöchentlicher Gabe. Die Beobachtungszeit dieser Studie ist jedoch sicher noch zu kurz um diese Frage endgültig zu beantworten.

Lück: Für Paclitaxel ist die wöchentliche Gabe die sinnvollste Dosierung, für Docetaxel möglicherweise auch (geringere Myelotoxizität, aber mehr Nagelprobleme).

Nitz: Nein, ich glaube dass sich aus Praktikabilitätsgründen PACS 01 durchsetzen wird.

Steger: Die vorliegenden Ergebnisse zur adjuvanten und neoadjuvanten dosisdichten Therapie sind nicht eindeutig interpretierbar. Daher sollte meines Erachtens diese Therapieform vorläufig weiter ausschließlich innerhalb kontrollierter Studien angeboten werden, so wie es die Konsensuskonferenz von St. Gallen 2005 auch empfiehlt. Es sind die entsprechenden Daten abzuwarten bevor eine Empfehlung abgegeben werden kann. Prinzipiell ist es ein attraktives Konzept, wobei die endgültige Bedeutung von den weiteren Daten abhängen wird.

Thomssen: Die wöchentliche sequentielle Paclitaxelapplikation ist eine attraktive Alternative. Sie ist aber sicher nicht die einzig belegte Applikationsform von Taxanen. Die US-Intergroup-Studie \#1199 hatte zum Ziel, die Effektivität der verfügbaren Taxane in der adjuvanten Therapiesequenz mit $4 \mathrm{Zy}$ klen AC gefolgt von 3 Monaten Taxantherapie direkt zu vergleichen. Es konnte in der Hauptfragestellung kein Unterschied zwischen den Substanzen gefunden werden. Als Nebenbeobachtung wurde im Trend eine etwas bessere Effektivität bei Einsatz von Docetaxel im 3-wöchentlichen Schema sowie bei Einsatz von Paclitaxel im dosisdichteren wöchentlichen Schema beobachtet. Die Studie GEICAM-9906 mit dosisdichter wöchentlicher Paclitaxel-Applikation stellt vor allem eine Bestätigung der französischen Studie PACS-01 dar, d.h. der Überlegenheit der FEC-Taxan-Sequenz gegenüber Standard-FEC. Die wöchentliche Gabe von Paclitaxel scheint dabei eine verträgliche Alternative $\mathrm{zu}$ sein, das Gesamtschema FEC-Paclitaxel ist aber um 6 Wochen länger als das PACS-01-Schema FEC-Docetaxel.

\section{Frage 6: Welchen dokumentierten Prozentsatz an febriler Neutropenie akzeptieren Sie, bevor Sie eine primäre Prophylaxe mit G-CSF oder Pegfilgrastim durchführen?}

Eiermann: Etwa 5-10\%.

Fridrik: Entsprechend den neuen Richtlinien der American Society of Clinical Oncology würde ich eine primäre Prophylaxe bei einer erwarteten Infektrate von über $20 \%$ durchführen. Dies würde alle taxanhaltigen adjuvanten Therapien betreffen und die Toxizität beträchtlich senken.

Lück: Maximal 5\%.

Nitz: Gemäß der neuen Leitlinie akzeptiere ich $20 \%$ febrile Neutropenie.

Steger: Entsprechend der adjuvanten Situation sollte bei beeinflussbaren bzw. vermeidbaren Risikosituationen wie sie die febrile Neutropenie darstellt, entsprechend Wert auf größtmögliche Patientinnensicherheit gelegt werden. Ich persönlich erachte $>10 \%$ als Indikation zur primären G-CSF-Prophylaxe. Thomssen: Grundsätzlich sollte gemäß NCCN-Leitlinien (NCCN - Practice Guidelines in Oncology - v.2.2005) ab einem Risiko von $10 \%$ schwerer oder febriler Neutropenie großzügig von einer Prophylaxe mit G-CSF oder Pegfilgrastim Gebrauch gemacht werden. Das Risiko für eine schwere oder febrile Neutropenie muss individuell in Abhängigkeit von der für die verschiedenen Chemotherapieschemata dokumentierten Häufigkeiten, aber auch von Alter, Allgemeinzustand, Vortherapien und Ausgangsleukozytenwert abgeschätzt werden.

\section{Frage 7: Dosisdichte Regime konnten eine Überlegen- heit gegenüber konventionellen Regimen zeigen (CALGB 9741, AGO ETC-trial). Setzen Sie in bestimm- ten Risikokonstellationen diese Regime auch außerhalb von Studien ein?}

Eiermann: Das AGO-ETC-trial-Regime setzen wir bei 4 und mehr befallenen Lymphknoten ein.

Fridrik: In der CALGB-Studie 9741 profitierten Patientinnen mit rezeptorpositiven und -negativen Mammakarzinomen von der dosisdichten Therapie. Bei den rezeptornegativen war der Unterschied signifikant. Das Konzept einer dosisdichten The- 
rapie bietet sich vor allem für rasch proliferierende Tumoren an. Rezeptorpositive Tumorzellen sind häufiger schlafende Zellen. Dosisdichte Therapie setzte ich bei Mammakarzinomen mit mehr als 3 positiven Lymphknoten und negativem Hormonrezeptor ein.

Lück: Ja, insbesondere bei $>4$ befallenen Lymphknoten.

Nitz: Ja, bei $\mathrm{N}+$, High-risk-Tumoren.

Steger: Nein, (siehe Frage 5). Außerhalb klinischer Studien ist diese Therapiekonzept meines Erachtens noch nicht zu empfehlen. Eine kürzlich publizierte Meta-Analyse, die keinen signifikanten Vorteil für das dosisdichte Konzept zeigte, weist auch in diese Richtung.

Thomssen: Für dosisdichte Chemotherapien wurde in mehreren Studien (CALGB-9741, AGO-Studie diETC vs EC-T) eine Überlegenheit gezeigt. Die Daten der Studie CALGB9741 wurden in San Antonio 2005 als Endanalyse vorgestellt. Unklar ist, ob dieser Vorteil nur für Patientinnen mit HR- Tumoren gilt. Ich würde bei nodal positiven Patientinnen außerhalb von Studien grundsätzlich die Möglichkeit einer dosisintensiven und taxanhaltigen Chemotherapie erwägen. Diese sollte am besten im Rahmen des GAIN-Protokolls der deutschen Intergroup AGO-GBG-NOGGO-Studie angeboten werden.

Frage 8: Erschwert die komplette Alopezie unter Taxanen nach Ihrer Einschätzung die Compliance der Patientin?

\section{Eiermann: Nein.}

Fridrik: Die starke Alopezie ist nach meiner Erfahrung sicher ein Problem für die meisten Patientinnen, hält aber nur wenige von der Entscheidung für ein taxanhaltiges Regime ab. Ein größeres Problem ist die Asthenie, auf Grund derer ein nicht unerheblicher Teil der Patientinnen die Therapie abbricht.

Lück: In der adjuvanten Situation erschwert die Alopezie die Compliance nicht.

Nitz: Nein.

Steger: Mit entsprechender Aufklärung und bei seriöser Risikoabwägung: nein.

Thomssen: Unsere Erfahrung zeigt, dass nach ausführlicher Aufklärung die Patientinnen im Allgemeinen sehr wohl die Bedeutung der adäquaten Chemotherapien verstehen und die Alopezie - nolens-volens - akzeptieren. Patientinnen, die eine Alopezie nicht akzeptieren, sind häufig auch vollständig gegen eine Chemotherapie eingestellt.

Frage 9: Erwarten Sie in der nächsten Zukunft die klinische Einführung von besser verträglichen und möglicherweise wirksameren Taxanen?

Eiermann: Nicht in der nahen Zukunft, in zirka 3-5 Jahren. Fridrik: Albumingebundene Paclitaxel-Nanopartikel (Nab-
Paclitaxel) sind in der metastasierten Situation auch bei taxanrefraktären Patientinnen wirksam. Das Problem ist die hohe Neurotoxizität. Interessanter sind aber die Epothilone. Lück: In den USA ist das albumingebundene Taxan bereits zugelassen.

Nitz: Ja, das ist zu erwarten.

Steger: Entsprechende klinische Phase-II/III-Studien, an denen wir auch beteiligt sind, laufen weltweit. Es ist zu hoffen, dass diese Substanzen einen noch höheren therapeutischen Index aufweisen als die beiden derzeit zur Verfügung stehenden Taxane.

Thomssen: Mehrere Firmen arbeiten intensiv an Weiterentwicklungen von Taxanen und taxanartigen Substanzen. Abgeschlossene Phase-III-Studien liegen derzeit für keine dieser Substanzen vor.

\section{Frage 10: Frauen mit einer Überexpression von HER2/neu sollte Trastuzumab (Herceptin ${ }^{\circledR}$ ) nicht vorenthalten werden. Mit welchem taxanhaltigen Regime kombinieren Sie den Antikörper bevorzugt?}

Eiermann: Aufgrund der Datenlage (US-Studien, BCIRG006) können beide Taxane eingesetzt werden mit einer nahezu identischen Risikoreduktion.

Fridrik: Mammakarzinome mit einer Überexpression des HER2/neu Rezeptors behandle ich bevorzugt neoadjuvant in der ABCSG Studie $24(6 \times$ Epirubicin/Docetaxel \pm Capecitabine), alle erhalten Trastuzumab entweder vor und nach der Operation oder nur postoperativ.

Lück: Epirubicin gefolgt von Paclitaxel.

Nitz: In Anlehnung an HERA; in Zukunft vielleicht simultan zu Taxan (Paclitaxel oder Docetaxel) nach $4 \times$ Epirubicin. Steger: Als eines der größten Zentren im Rahmen der HERAStudie favorisieren wir derzeit die sequentielle Herceptintherapie nach Chemotherapie. Auch hier erscheint bei entsprechender Indikation das sequentielle FEC100-Docetaxel-Regime gefolgt von Herceptin alle 3 Wochen für ein Jahr sehr praktikabel. Ebenso setzen wir sequentielles Herceptin nach neoadjuvanter Therapie mit 6-8 Zyklen eines Epirubicin-Docetaxel-Regimes ein.

Thomssen: Eine adjuvante Therapie mit dem Antikörper Trastuzumab (Herceptin) ist heutzutage Standard bei Patientinnen mit HER2/neu-überexprimierendem Mammakarzinom. Dies gilt für das nodal positive Mammakarzinom generell und für das nodal negative Karzinom bei Tumorgröße $>1$ $\mathrm{cm}$. Trastuzumab erhalten Patientinnen bei uns entsprechend der Häufigkeit der Chemotherapieschemata, die meist im Rahmen klinischer Studien gegeben werden: FEC-Doc, diETC, diEC-diTX, im neoadjuvanten Rahmen mit EC-Docetaxel und FEC-Paclitaxel. Eine Präferenz für ein bestimmtes Schema bei HER2/neu-überexprimierenden Karzinomen haben wir nicht. 
Frage 11: Wäre die beschriebene Kardiotoxizität unter Herceptin nach anthrazyklinhaltiger Chemotherapie für Sie ein Grund, verstärkt das TCH-Regime (Docetaxel, Platin, Herceptin) zu berücksichtigen?

Eiermann: Ja, insbesondere bei kardialen Risikopatientinnen, in Zukunft vielleicht auch bei TOP II negativen Patientinnen. Fridrik: In einer randomisierten Studie der BCIRG wurden 3 adjuvante Therapiearme bei HER2/neu-überexprimierenden Mammakarzinomen untersucht. Doxorubicin/Cyclophosphamid gefolgt von Docetaxel versus Doxorubicin/Cyclophosphamid gefolgt von Docetaxel und Trastuzumab versus Carboplatin/Docetaxel/Trastuzumab. Beide trastuzumabhaltigen Regime waren gleich wirksam, das Anthracyclinregime aber wesentlich toxischer. Der Beobachtungszeitraum ist aber noch zu kurz, um von einer identischen Wirksamkeit des nicht anthracylinhaltigen Regime auszugehen. Ich würde daher TCH nur bei einer Kontraindikation gegen Anthracyclin verwenden.

Lück: Sicher.

Nitz: Nur in Ausnahmefällen z.B. grenzwertige LVEF, hohes Alter.

Steger: Wenn die Überlebensdaten für das TCH-Regime entsprechend ausfallen, ja.

Thomssen: Die Kombination Docetaxel-Carboplatin-Trastuzumab ist ein hochinteressanter Therapieansatz. In der klinischen Studie BCIRG-006 konnte die Überlegenheit dieser Kombination gegenüber dem trastuzumabfreien Standardarm gezeigt werden, aber nicht gegen die trastuzumab- und anthrazyklinhaltige Standardkombination. Aufgrund der offensichtlich geringeren kardialen Ereignisse könnte dieses Schema insbesondere bei Patientinnen mit kardialem Risiko in Erwägung gezogen werden. Ob dieses Schema bei kardialer Vorgeschichte wirklich unbedenklich ist, ist allerdings nicht untersucht. Interessant ist sicher der Einsatz dieser Kombina- tion, wenn den Tumoren eine zusätzliche Topoisomerase-2 $\alpha$ Expression fehlt. Bei Topoisomerase- $2 \alpha$-Überexpression ist die anthrazyklinhaltige Kombination überlegen.

\section{Teilnehmer}

Prof. Dr. Wolfgang Eiermann

BRK Schwesternschaft, Gesundheitszentrum München gGmbH Frauenklinik vom Roten Kreuz

Taxisstrasse 3

80637 München, Deutschland

Doz. Dr. Michael Fridrik

Allgemeines Krankenhaus Linz

Vorstand der Abteilung Onkologie

Krankenhausstr. 9

4021 Linz, Österreich

Prof. Dr. Hans-Joachim Lück

Dr.-Horst-Schmidt-Kliniken

Klinik für Gynäkologie und gynäkologische Onkologie

Ludwig-Erhard-Str. 100

65199 Wiesbaden, Deutschland

Prof. Dr. Ulrike Nitz

Universitäts-Frauenklinik

Moorenstraße 5

40225 Düsseldorf, Deutschland

Univ.-Prof. Dr. Günther Steger

Medizinische Universität Wien

Klinische Abteilung für Onkologie

Währinger Gürtel 18-20

1090 Wien, Österreich

Prof. Dr. Christoph Thomssen

Martin-Luther-Universität Halle-Wittenberg

Brustzentrum Halle

Ernst-Grube-Str. 40

06097 Halle/Saale, Deutschland 